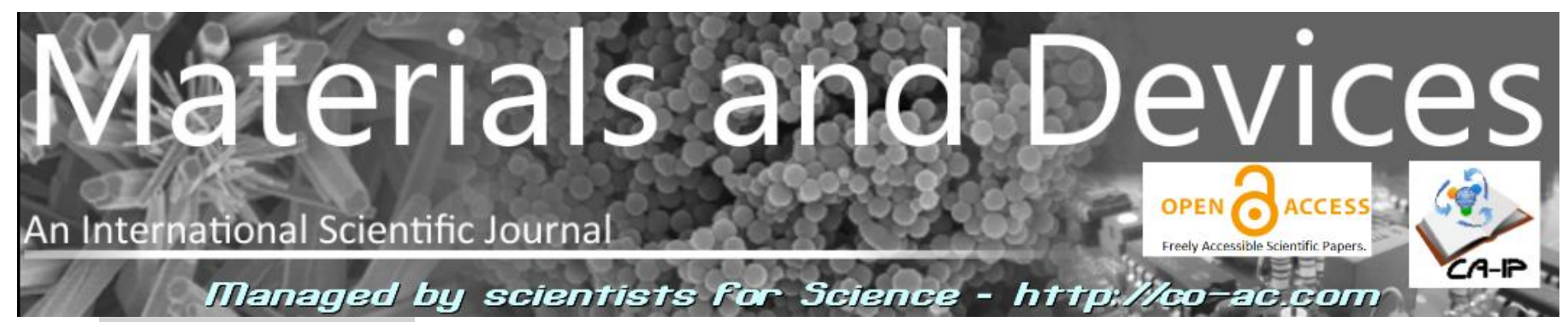

Article type: Conf.- Invited conference paper (ISPDS2)

\title{
Carbon nanotubes functionalized with copper hexacyanoferrate nanoparticles for a selective extraction of cesium from liquid waste
}

H. Draouil (1,2,3,4) L. Alvarez (4), J. Causse (1), V . Flaud (5), M.A. Zaïbi (3), J.L. Bantignies (4), M. Oueslati (2) and J. Cambedouzou (1)

(1) Institut de Chimie Séparative de Marcoule, CEA-CNRS-ENSCM-UM, 30207 Bagnols sur Cèze, France

(2) Unité de nanomatériaux et photonique, Université El Manar, Faculté des Sciences de Tunis, Département de Physique, 2092 El Manar, Tunis Tunisia

(3) Ecole Nationale Supérieure d'Ingénieurs de Tunis, Université de Tunis, 1008 Tunis, Tunisia

(4) Laboratoire Charles Coulomb, CNRS, Université de Montpellier, F-34095 Montpellier, France

(5) Institut Charles Gerhardt Montpellier, Université de Montpellier, F-34095 Montpellier, France

\section{Corresponding author : laurent.alvarez@umontpellier.fr}

RECEIVED: 03 May 2018 / RECEIVED IN FINAL FORM: 01 July 2018 / ACCEPTED/: 02 July 2018

\begin{abstract}
Single-walled carbon nanotubes are functionalized with copper hexacyanoferrate nanoparticles for the liquid-solid extraction of cesium from liquid waste and contaminated water. The functionalization process is followed mainly by $\mathrm{x}$-ray photoemission spectroscopy. Indeed, determining the chemical environment around carbon or nitrogen atoms allows to evidence the formation of covalent bounding. In addition, the signatures of iron and copper ions give information on the effective growth of hexacyanoferrate nanoparticles. Furthermore, the cesium sorption mechanism is investigated by comparing the peak intensities associated to the response of potassium and cesium ions. Finally, based on the liquid chromatography analyzes, the sorption of cesium with the functionalized carbon supports is studied. The main results of this work are the demonstration of both a good selectivity of cesium trapping and a high sorption capacity by hybrid single-walled carbon nanotubes.
\end{abstract}

Keywords : LIQUID WASTE, NANOTUBES, HEXACYANOFERRATE, FUNCTIONALIZATION, CESIUM TRAPPING 


\section{Introduction}

Decontamination of radioactive water, whether from nuclear effluents from industry and research, or from nuclear accidents, is a major challenge to limit releases to the environment [1-2]. In particular, radioactive iodine (I-131) and cesium (Cs-137) are particularly toxic and found in large quantities in polluted waters [3-4]. It therefore becomes urgent to provide sustainable solutions for the treatment of contaminated water, in particular with radioactive cesium ions $\left(\mathrm{Cs}^{+}\right)$.

Several materials have been proposed for this application, but the frequent coexistence of other ions whose concentration is higher than Cs+ limits the efficiency of decontamination. Thus, the use of selective sorbents is absolutely mandatory. Prussian blue analogues have been shown to be particularly relevant for this application. In particular, they are useful for the treatment of contaminated natural water or diluted wastes with typical Cs+ concentrations ranging from $10^{-9} \mathrm{~g}$ $\mathrm{L}^{-1}$ for contaminated water and up to $10^{-4} \mathrm{~g} \mathrm{~L}^{-1}$ for low to intermediate level wastes [4]. However, once hydrated, this material forms sludge that is difficult to reprocess afterwards, which makes difficult its utilization [5-6]. Then, Prussian blue grafting tests on mesoporous silica have been carried out. This allows a continuous flowing on solid support, affording the treatment of large quantities of water. However this latter material exhibits a low sorption rate and has a weakness inherent in its chemical composition towards aggressive media such as basic medium [7-8].

Single walled-carbon nanotubes are solid materials displaying a high specific area, good mechanical and chemical resistances. However, pristine nanotubes (including multiwalled) exhibit a poor sorption capacity [9-10]. Outer surface functionalization with dimethyl-acetylene dicarboxylate increase significantly the whole sorption capacity but without any selectivity [11].

Consequently, in order to overcome all these drawbacks, this work is focused on the hexacyanoferrate nanoparticles (HCF, which are Prussian blue analogs) grafting onto single-walled carbon nanotubes (NT) in order to elaborate new hybrid system (HFC-NT) for a selective cesium extraction with high capacity.

\section{Experimental section}

Nanotubes are purchased from Carbon Solution, Inc.(reference P2-SWCNTs, of diameter $1.4 \pm 0,2 \mathrm{~nm}$ ). Propargylamine (PRG) and Copper-hexacyanoferrate precusors are purchased from Sigma-Aldrich (99\%) and used without further purification.

\section{1- Functionalization with propargylamine}

A mixture containing $60 \mathrm{mg}$ of $\mathrm{NT}$ with $25 \mathrm{ml}$ of pure PRG is prepared. The mixture is submitted to ultrasonication for 15 min following the procedure described elsewhere [10]. The mixture is then transferred into a teflon-lined steel autoclave and heated to $100^{\circ} \mathrm{C}$ during 24 hours. This blend is filtered and then washed with acetone in order to remove all PRG molecules in excess.

\section{2- Cu-HFC-NPs growth}

This step is based on two reactions, following the procedure described in a previous work [11]. The first step features the addition of a copper nitrate $\left(\mathrm{Cu}\left(\mathrm{NO}_{3}\right)_{2}\right)$ solution in order to attach a $\mathrm{Cu}$ atom to the PRG. This $\mathrm{Cu}$ atom serves as a nucleation center for the further growth of the CuHCF-NP. The resulting system is carefully rinsed with acetone. The second step consists in the addition of a potassium ferrocyanide $\left(\mathrm{K}_{4} \mathrm{Fe}(\mathrm{CN})_{6}\right)$ solution aimed at initiating the growth of the first cell of the CuHCF-NP. Both solutions are prepared with a concentration of $10^{-2} \mathrm{M}$. This process is cycled three to four times.

\section{4- Characterization}

$X$-ray photoelectron spectroscopy (XPS) is performed on an ESCALAB 250 (Thermo Electron). The X-ray excitation is provided by a monochromatic AI Ka $(1486.6 \mathrm{eV})$ source. All XPS spectra are treated using the AVANTAGE software.

Liquid phase ionic chromatography (LPIC) is performed using an ICS-5000-Dionex instrument with 4 scans for each sample.

\section{5- Cesium sorption}

Cs+ sorption measurements are carried out in batch mode on both pristine NT and on HCF-NT according to the following protocol: $10 \mathrm{mg}$ of a buckypaper of carbon nanotubes are stirred with $20 \mathrm{~mL}$ of cesium nitrate solution using different concentrations ( $0.1 \mathrm{mM}, 0.5 \mathrm{mM}, 2 \mathrm{mM}, 4 \mathrm{mM}$ and $8 \mathrm{mM})$ for $24 \mathrm{~h}$. Carbon nanotubes are recovered by filtration using filter paper with particle retention between 5 and $13 \mathrm{~mm}$ and thoroughly washed using Milli-Q water. The solid samples are then dried for further analyses, while the liquid phase is analyzed by LPIC.

\section{Functionalization processes}

The different functionalization steps are investigated by XPS. Figure 1 displays the low resolution spectra for pristine nanotube (black curve, 1), PRG grafted on NT (red curve, 2) and HCF-NT (blue curve, 3). For pristine NT, two chemical elements are identified: carbon atoms (peak around $285 \mathrm{eV}$ ) of the nanotubes and some oxygen (530 eV) probably covalently bond to the carbon network, giving rise to defects [12].

After PRG ( $\left.\mathrm{H}-\mathrm{C} \equiv \mathrm{C}-\mathrm{CH}_{2}-\mathrm{NH}_{2}\right)$ grafting, a new peak at around 400 $\mathrm{eV}$ is observed, featuring the presence of nitrogen [13]. After HCF nanoparticle growth $\mathrm{K}_{2} \mathrm{CuFe}(\mathrm{CN})_{6}$, new and expected peaks assigned to potassium (295 eV), iron (730 eV) and copper (932 $\mathrm{eV}$ ) appear in the photoemission spectrum (fig.1.c) [14,15]. To better investigate the functionalization process and the chemical bounding formed, high resolution measurements are required.

Figure 2 displays the deconvoluted $\mathrm{C} 1 \mathrm{~s}$ peak at each 
functionalization step. Comparing the $\mathrm{C} 1 \mathrm{~s}$ peaks of pristine NTs (fig.2.a) with PRG grafted NT samples (fig.2.c), we clearly see changes around $286 \mathrm{eV}$ (linked to $\mathrm{CO}$ or CN bonds [12]), especially in terms of full width at half maximum (FWHM) ( $1 \mathrm{eV}$ for pristine NT and $1.3 \mathrm{eV}$ for PRG-NT).

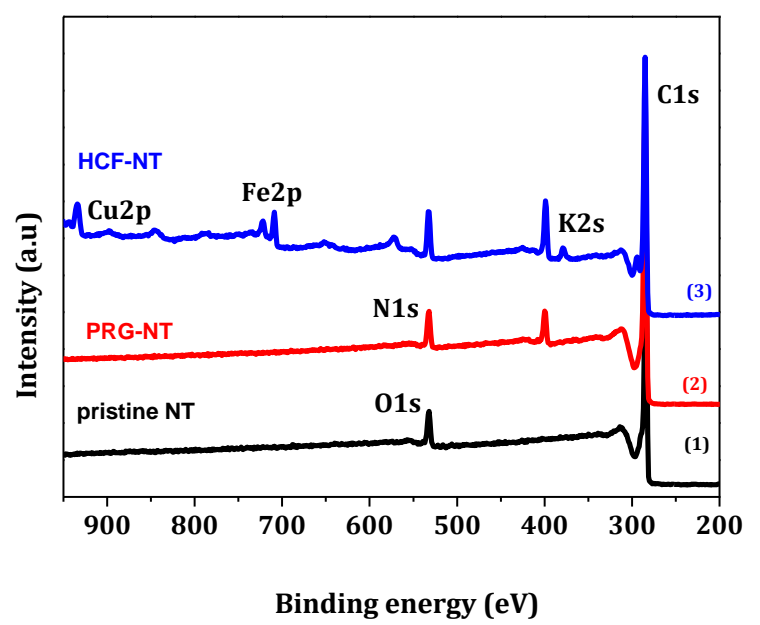

Figure 1: XPS survey at each grafting step

Quantitatively, this contribution has significantly increased (from $4 \%$ up to $\sim 14 \%$ ). This evolution is concomitant with a decrease of the sp2 peak at $284.4 \mathrm{eV}$ and an increase of the sp3 contribution at $285 \mathrm{eV}$. Indeed, NT functionalization with PRG leads to a change of sp2 orbitals into sp3 orbitals in order to ensure a covalent bonding between the carbon atoms of the NT and PRG. In addition, the $\mathrm{C}-\mathrm{N}$ bondings of the PRG molecules increase significantly the peak intensity at around $286 \mathrm{eV}$ which can arise either from C-O or C-N (and even triple bonding between carbon atoms) [12]. For NT functionalized with HCF (Fig. 2.c), the signal around $286 \mathrm{eV}$ (relative to $\mathrm{C}-\mathrm{N}, \mathrm{C}-\mathrm{O}$ or $\mathrm{C} \equiv \mathrm{C}$ bonds) decreases with respect to the PRG-NT (from 14\% to $10.6 \%$ ). One possible assumption is to consider that the washing step after HCF functionalization- that is to say after the growth of hexacyanoferrate nanoparticles, PRG-CuHCF particles not covalently bound to NT could have been removed, which induces a decrasing of $\mathrm{C} \equiv \mathrm{C}$ bond signal.

Typical binding energy for nitrogen atoms in cyanide groups of hexacyanoferrate molecules is between $397.5 \mathrm{eV}$ and 400 $\mathrm{eV}$ [15]. Figure 3 shows the high resolution N1s spectra of PRG-NT (fig. 3.a) and HCF-NT (fig. 3.b) samples. The most important signal at around $399 \mathrm{eV}$ is due to $\mathrm{C}-\mathrm{N}$ bonding from the PRG molecules whereas the peaks at around 400 et 402 $\mathrm{eV}$, assigned to $\mathrm{N}-\mathrm{C}=\mathrm{O}$ and nitrogen ions correspond to defects and contamination after the different chemical treatments. Following the growth of HCF nanoparticles, a new peak appears around $397.6 \mathrm{eV}$, assigned to the $\mathrm{C} \equiv \mathrm{N}$ bonds expected in HCF nanoparticles [15].

Figures 4 and 5 display peaks at 708.3 and $932.7 \mathrm{eV}$ corresponding to Fe2p and Cu2p binding energies [14-15]. The Fe2p energy is in good agreement with the formation of $\mathrm{Fe}^{2+}$, as expected in case of copper HCF. Biesinger et al [14] analyzed different types of copper samples such as $\mathrm{Cu}$ and $\mathrm{Cu}_{2} \mathrm{O}$, and found the same Cu2p decomposition profile as in our sample.

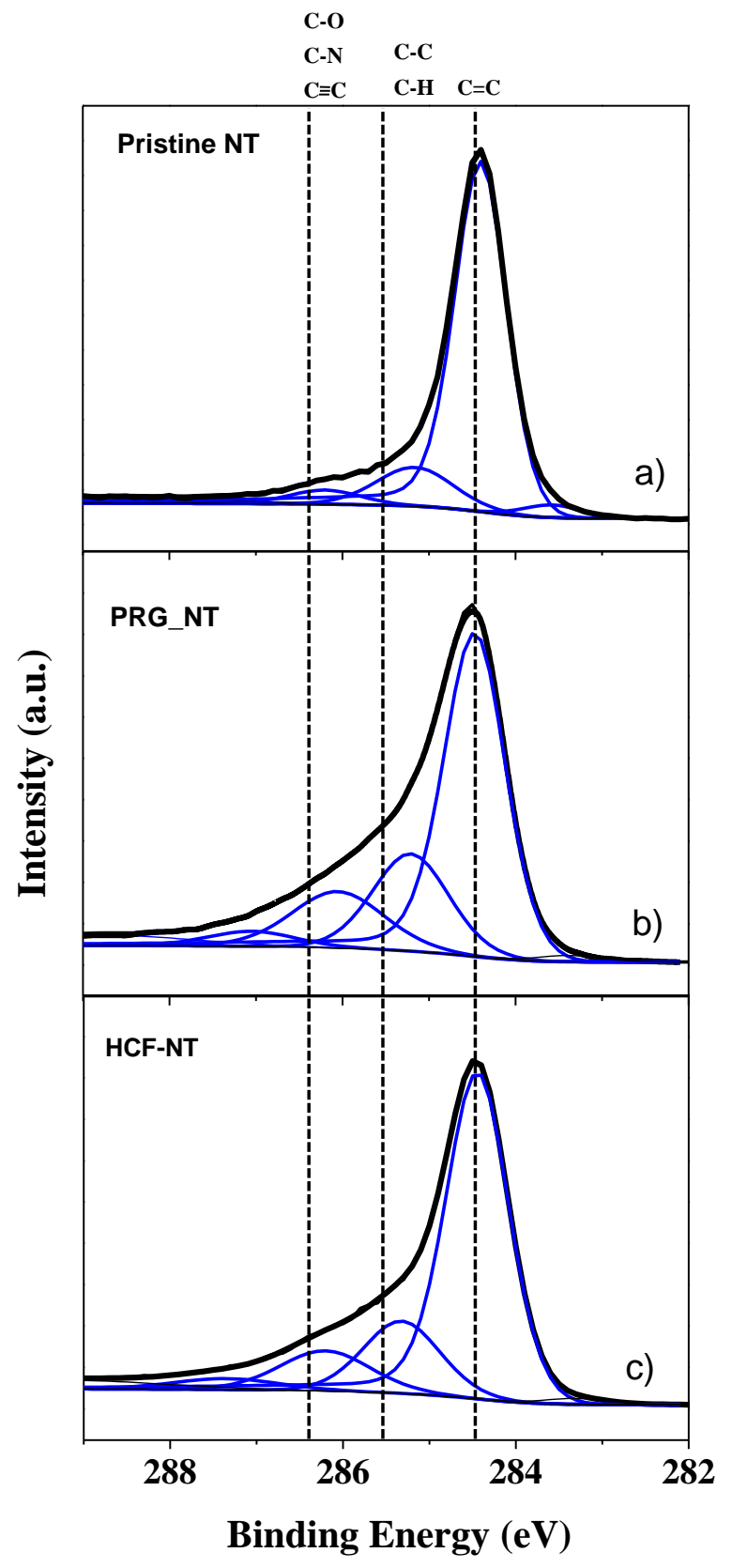

Figure 2: High resolution C1s x-ray photoemission spectra after each grafting step

They attributed the lower energy bond to $\mathrm{Cu}^{+}$and the higher to $\mathrm{Cu}^{2+}$. In these compounds, $\mathrm{Cu}^{2+}$ is generally expected [15-16], but a reversible reduction reaction in $\mathrm{Cu}^{+}$is sometimes reported [17]. It is worth mentioning that the intensity ration between 
the two peaks depend on the area investigated.

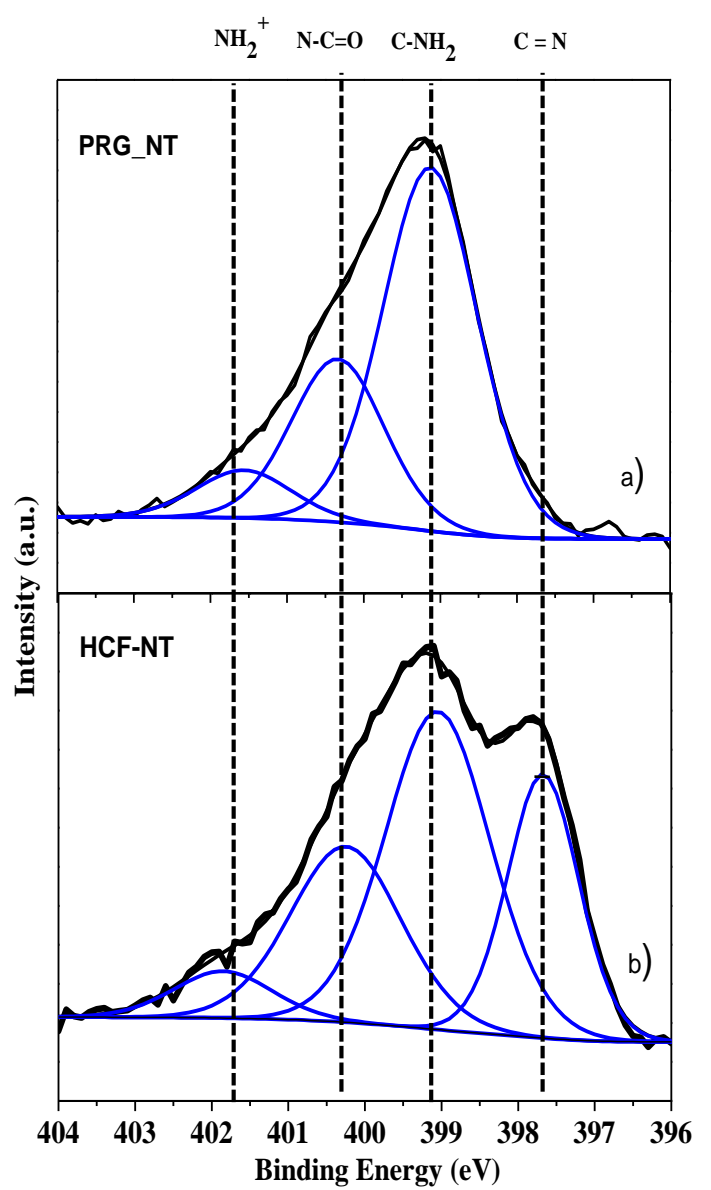

Figure 3: High resolution N1s x-ray photoemission spectra after each grafting step

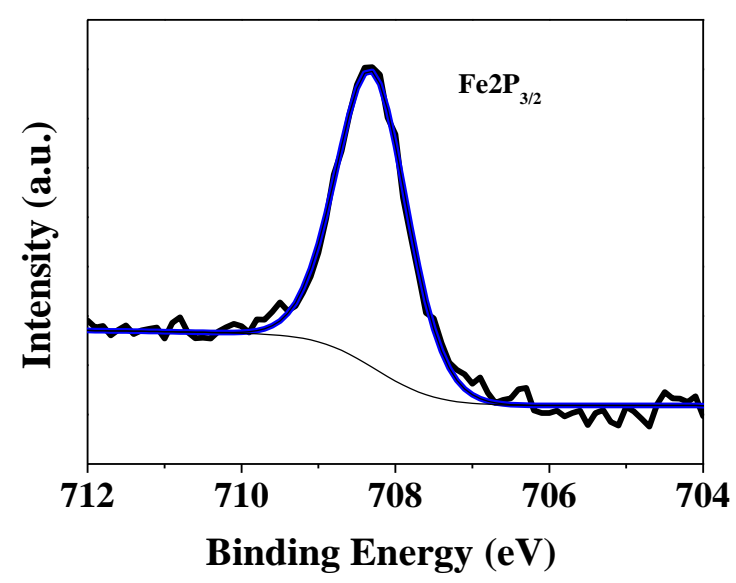

Figure 4: High resolution Fe2p x-ray photoemission spectrum of HCF-NT
It should be noted that the ratio $\mathrm{Cu}^{+} / \mathrm{Cu}^{2+}$ strongly depends on the zone studied in our samples. As we do not believe in the heterogeneity of our samples, we assume a reduction under $\mathrm{X}$ rays, leading to a transformation from $\mathrm{Cu}^{2+}$ to $\mathrm{Cu}^{+}$. Anyway, all the results are consistent with an efficient process of functionalization.

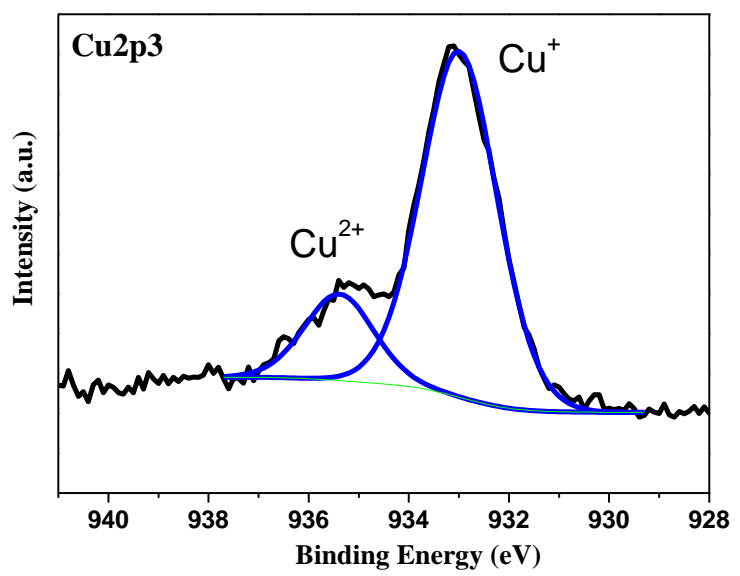

Figure 5: High resolution Cu2p x-ray photoemission spectrum of HCF-NT.

\section{Cesium sorption capacity}

The $\mathrm{Cs}^{+}$sorption measurements are carried out on both pristine and functionalized nanotubes with HFC.

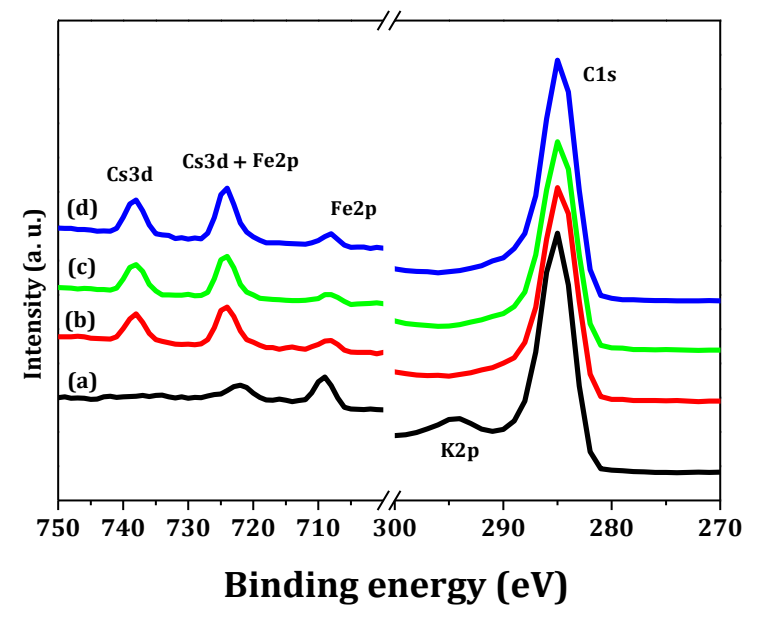

Figure 6: XPS survey for pristine HCF-NT (a) and exposed to solutions containing different $\mathrm{Cs}$ concentrations (b) 0.1 $\mathrm{mM}$, (c) $2 \mathrm{mM}$, (d) $4 \mathrm{mM}$ )

The XPS surveys of NT functionalized by HFC before and after exposure to $\mathrm{CSNO}_{3}$ solutions of different Cs concentrations 
$(0.1 \mathrm{mM}, 2 \mathrm{mM}$ and $4 \mathrm{mM})$ are presented in Figure 6. The presence of $\mathrm{Cs}$ is detected in two positions of binding energy: $\sim 738 \mathrm{eV}$ and $~ 724 \mathrm{eV}$ [18] (Fig. b, c and d). The intensity of these peaks increases with the concentration of $\mathrm{CsNO}_{3}$. Concomitantly, the disappearance of the signal from potassium is observed. This result confirms an exchange between $\mathrm{Cs}+$ and $\mathrm{K}^{+}$ions within the HFC cubic structure. Finally, we observe $\mathrm{Cs}^{+}$XPS signal that increases very slowly, suggesting that sites available for $\mathrm{Cs}^{+}$sorption are rapidly saturated.

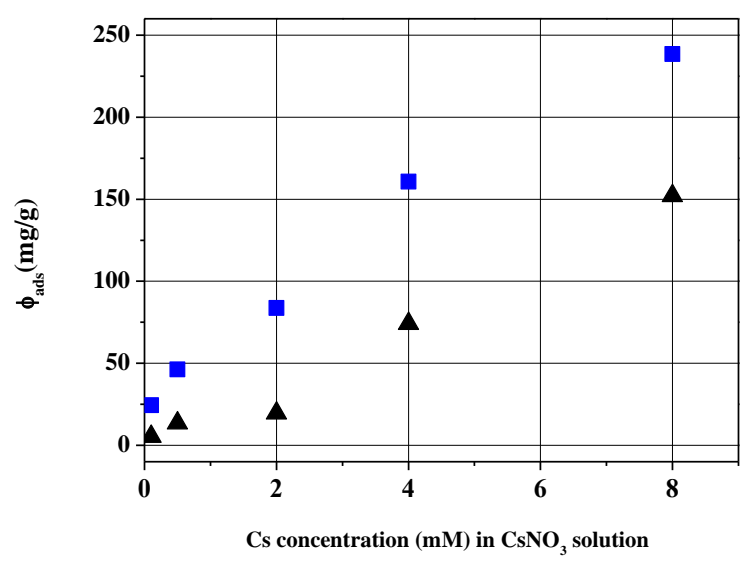

Figure 7: Sorption isotherm of Cs+ on NT and HCF-NT

In order to go further in understanding the sorption mechanism, we also conducted LPIC analyzes of the cesium solution before and after exposure to HFC-NT. The $\mathrm{Cs}^{+}$ sorption isotherm by pristine and functionalized nanotubes with HFC are shown in figure 7. Both NT materials show increasing sorption capacity with increasing concentration of $\mathrm{Cs}^{+}$, although it is still lower for pristine NT with respect to HCF-NT (150 mg.g1 and $230 \mathrm{mg} \cdot \mathrm{g}^{-1}$ respectively). One way to evaluate the proportion of $\mathrm{Cs}^{+}$ions complexed in HFC nanoparticles (i.e. selectively trapped) is to subtract the sorption isotherm from the pristine NT to that of HFC. Thus, for Cs concentration of $4 \mathrm{mM}$, the selective sorption of Cs reaches a value of about 80 $\mathrm{mg} \cdot \mathrm{g}^{-1}$ (about a third of the total sorption of the HFC-NT). This value compares rather well with other materials generally used for Cs extraction.

\section{Conclusions}

Hexacyanoferrate nanoparticules (prussian blue analogs) have been successfully grafted at the outer surface of carbón nanotubes using propargylamine molecules as intermediate species. The different steps of the whole fuunctionalization process have been investigated mainly by means of $x$-ray photoemission spectroscopy. The study of the different chemical environments around the carbón and nitrogen atoms allowed to evidence the formation of the expected covalent bondings at each step of the process. The nature of iron and copper ionic species confirms the growth of copper hexacyanoferrate nanoparticules. Cesium sorption capacity has been studied by liquid phase ionic chromatography. The reached value of 80 $\mathrm{mg} / \mathrm{g}$ is particularly encouraging. Finally, ion exchange mechanism between potassium and cesium species has been confirmed by photoemission measurements.

\section{REFERENCES}

1. W.-Q. Shi, L.-Y. Yuan, Z.-J. Li, J.-H. Lan, Y.-L. Zhao and Z.-F. Chai, Radiochimica. Acta, vol.100, p 727 (2012)

2. P. P. Povinec, M. Aoyama, D. Biddulph et al, Biogeosciences, vol.10, p 5481, (2013)

3. D. Yang, S. Sarina, H. Zhu, H. Liu, Z. Zheng, M. Xie, S.V. Smith and S. Komarneni, Angewandte Chemie International Edition, vol.50, p 10594 (2011)

4. K. Hirose, Journal of Environmental Radioactivity, vol.157, p 113 (2016)

5. J. Robertson, Materials Science and engineering, R37, p 129 (2002)

6. F. P. Bundy, H. T Hall, H. M strong, R. H. Wentorf, Nature, vol.176, p 51 (1955)

7. H. Nishijima, S. Akita, Y. Nakayama, K. I. Hohmura, S. H. Yoshimura, K. Takkaeyasu, App Phys Lett, 74, p 4060 (1999)

8. J. D. Bernal, Proceeding of the royal scociety, A106, p 749 (1924)

9. R. Yavari, Y. D. Huang and S. J. Ahmadi, Journal of Radioanalytical Nuclear Chemistry, vol.287, p 393 (2011)

10. H. Kaper, J. Nicolle, J. Cambedouzou and A. Grandjean, Materials Chemistry and Physics, vol.147, p 147 (2014) 
11. A. Grandjean, C. Delchet, J. Causse, Y. Barre', Y. Guari and J. Larionova, Journal of Radioanalytical Nuclear Chemistry., vol.307, p 427 (2016)

12. R. Larciprete, P. Lacovig, S. Gardonio, Al. Baraldi, and Silvano Lizzit, Journal of Physical Chemistry. C, vol.116, p 9900 (2012)

13. P. Chambrion, T. Suzuki, Z-G. Zhang, T. Kyotani, and A. Tomita Energy \& Fuels, vol.11, p 681 (1997)

14. M. C. Biesinger, L. W.M. Lau, A. R. Gerson, R. St.C. Smart, Applied Surface Science, vol.257, p 887 (2010)

15. A. Lisowska-Oleksiak, M. Wilamowska, V. Jasulaitiené, Electrochimica Acta, vol.56, p 3626 (2011)

16. G. Yang,Y. Shen, Mi. Wang, H. Chen,B. Liu. S. Dong, Talanta, vol.68, p 741 (2006)

17. O. Makowski, J. Stroka, P.J. Kulesza, M.A. Malik, Z. Galus, Journal of Electroanalytical Chemistry, vol.532, p 157 (2002)

18. R. Chen, Hi. Tanaka, T. Kawamoto, M. Asai, C. Fukushima et al, ACS Applied Materials and Interfaces, vol.5, p 12984 (2013) 
Hajer Draouil et al, carbon nanotubes functionalized with copper hexacyanoferrate

Important: Articles are published under the responsability of authors, in particular concerning the respect of copyrights. Readers are aware that the contents of published articles may involve hazardous experiments if reproduced; the reproduction of experimental procedures described in articles is under the responsability of readers and their own analysis of potential danger.

\section{Reprint freely distributable - Open access article}

Materials and Devices is an Open Access journal which publishes original, and peer-reviewed papers accessible only via internet, freely for all. Your published article can be freely downloaded, and self archiving of your paper is allowed and encouraged!

We apply « the principles of transparency and best practice in scholarly publishing » as defined by the Committee on Publication Ethics (COPE), the Directory of Open Access Journals (DOAJ), and the Open Access Scholarly Publishers Organization (OASPA). The journal has thus been worked out in such a way as complying with the requirements issued by OASPA and DOAJ in order to apply to these organizations soon.

Copyright on any article in Materials and Devices is retained by the author(s) under the Creative Commons (Attribution-

NonCommercial-NoDerivatives 4.0 International (CC BY-NC-ND 4.0)), which is favourable to authors.

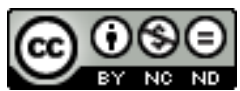

Aims and Scope of the journal : the topics covered by the journal are wide, Materials and Devices aims at publishing papers on all aspects related to materials (including experimental techniques and methods), and devices in a wide sense provided they integrate specific materials. Works in relation with sustainable development are welcome. The journal publishes several types of papers : A: regular papers, $L$ : short papers, $R$ : review papers, $\mathrm{T}$ : technical papers, Ur : Unexpected and « negative » results, Conf: conference papers.

$$
\text { (see details in the site of the journal: http://materialsanddevices.co-ac.com) }
$$

We want to maintain Materials and Devices Open Access and free of charge thanks to volunteerism, the journal is managed by scientists for science! You are welcome if you desire to join the team!

Advertising in our pages helps us! Companies selling scientific equipments and technologies are particularly relevant for ads in several places to inform about their products (in article pages as below, journal site, published volumes pages, ...). Corporate sponsorship is also welcome! 